\title{
On the Boundary Conditions in Deformed Quantum Mechanics with Minimal Length Uncertainty
}

\author{
Pouria Pedram \\ Department of Physics, Science and Research Branch, Islamic Azad University, Tehran 1477893855, Iran \\ Correspondence should be addressed to Pouria Pedram; p.pedram@srbiau.ac.ir
}

Received 25 September 2013; Revised 27 November 2013; Accepted 28 November 2013

Academic Editor: Frederik G. Scholtz

Copyright (c) 2013 Pouria Pedram. This is an open access article distributed under the Creative Commons Attribution License, which permits unrestricted use, distribution, and reproduction in any medium, provided the original work is properly cited.

\begin{abstract}
We find the coordinate space wave functions, maximal localization states, and quasiposition wave functions in a GUP framework that implies a minimal length uncertainty using a formally self-adjoint representation. We show how the boundary conditions in quasiposition space can be exactly determined from the boundary conditions in coordinate space.
\end{abstract}

\section{Introduction}

The existence of a minimal length uncertainty proportional to the Planck length $\ell_{P} \sim 10^{-35} \mathrm{~m}$ is one of the common predictions of various candidates of quantum gravity. This idea in the context of the Generalized (Gravitational) Uncertainty Principle (GUP) has attracted much attention in recent years and many papers have appeared in the literature to address the effects of this minimal length on various quantum mechanical systems [1-14].

It is pointed out by Mead that gravity amplifies the Heisenberg's measurement uncertainty which makes it impossible to measure distances more accurate than Planck's length [15]. In fact, since the increase of the energies to probe small distances considerably disturbs the spacetime structure because of the gravitational effects, the spatial uncertainty eventually increases at energy scales as large as the Planck scale. This minimal length can be considered as a fundamental property of quantum spacetime, a natural UV regulator, and a solution for the trans-Planckian problem. Since the string theory with large or warped extra dimensions can lower the Planck scale into the TeV range, this fundamental length scale also moved into the reach of the Large Hadron Collider.

The thought experiments that support the minimal length proposal include the Heisenberg microscope with Newtonian gravity and its relativistic counterpart [15], limit to distance measurements [16], limit to clock synchronization, and limit to the measurement of the blackhole horizon [4]. Moreover, different approaches to quantum gravity such as string theory, loop quantum gravity, and loop quantum cosmology, quantized conformal fluctuations $[17,18]$, asymptotically safe gravity [19], and noncommutative geometry all indicate a fundamental limit to the resolution of structure.

Based on the Heisenberg's microscope and taking into account both the normal and the gravitational uncertainties one finds [20]

$$
\Delta X \gtrsim \frac{1}{2 \Delta P}+G \Delta P
$$

where $G=\ell_{P}^{2}$ is the gravitational constant. As Adler and Santiago observed this GUP is invariant under $\ell_{P} \Delta P \leftrightarrow 1 / \ell_{P} \Delta P$ and therefore has a momentum inversion symmetry. Because of the universality of the gravity, this correction modifies all Hamiltonians for the quantum systems near the Planck scale.

Recently, an experimental scheme is suggested by Pikovski et al. to test the presence of the minimal length scale in the context of quantum optics [21]. They used quantum optical control and optical interferometric techniques for direct measurement of the canonical commutator deformations of a massive object. This experiment does not need the Planck-scale accuracy of position measurement and can be reached by the current technology. Some attempts have been also made to test possible quantum gravitational phenomena using astronomical observations [22, 23].

In this paper, we consider a GUP that implies a minimal length uncertainty proportional to the Planck length. We find 
the exact coordinate space wave functions and quasiposition space wave functions using a formally self-adjoint representation. We first obtain the eigenfunctions of the position operator and the maximal localization states. Then we discuss how the boundary conditions can be imposed consistently in both coordinate space and quasiposition space.

\section{The Generalized Uncertainty Principle}

Consider the following one-dimensional deformed commutation relation [13]:

$$
[X, P]=i \hbar\left(1+\beta P^{2}\right)
$$

where for $\beta=0$ we recover the well-known commutation relation in ordinary quantum mechanics and $\Delta X \Delta P \geq$ $(\hbar / 2)\left(1+\beta(\Delta P)^{2}\right)$. Since $\Delta X$ cannot be made arbitrarily small, the absolutely smallest uncertainty in positions for this GUP is $(\Delta X)_{\min }=\hbar \sqrt{\beta}$. To proceed further, consider the following representation [11]:

$$
\begin{gathered}
X=x, \\
P=\frac{\tan (\sqrt{\beta} p)}{\sqrt{\beta}},
\end{gathered}
$$

which exactly satisfies (2). This representation is formally selfadjoint subject to the inner product:

$$
\langle\psi \mid \phi\rangle=\int_{-\pi / 2 \sqrt{\beta}}^{+\pi / 2 \sqrt{\beta}} \mathrm{d} p \psi^{*}(p) \phi(p)
$$

and preserves the ordinary nature of the position operator.

The operator $A$ with dense domain $\mathscr{D}(A)$ is self-adjoint if $\mathscr{D}(A)=\mathscr{D}\left(A^{\dagger}\right)$ and $A=A^{\dagger}$. However, for the position operator $X$ in the momentum space, we have

$$
\begin{aligned}
& \int_{-\pi / 2 \sqrt{\beta}}^{+\pi / 2 \sqrt{\beta}} \mathrm{d} p \psi^{*}(p)\left(i \hbar \frac{\partial}{\partial p}\right) \phi(p) \\
& =\int_{-\pi / 2 \sqrt{\beta}}^{+\pi / 2 \sqrt{\beta}} \mathrm{d} p\left(i \hbar \frac{\partial \psi(p)}{\partial p}\right)^{*} \phi(p)+\left.i \hbar \psi^{*}(p) \phi(p)\right|_{p=+\pi / 2 \sqrt{\beta}} \\
& \quad-\left.i \hbar \psi^{*}(p) \phi(p)\right|_{p=-\pi / 2 \sqrt{\beta}},
\end{aligned}
$$

where $\phi(p)$ vanishes at $p= \pm \pi / 2 \sqrt{\beta}$ and $\psi^{*}(p)$ takes arbitrary values at the boundaries. Indeed, the adjoint of the position operator $X^{\dagger}=i \hbar \partial / \partial p$ has the same form but it acts on a different space of functions

$$
\begin{aligned}
\mathscr{D}(X)=\left\{\phi, \phi^{\prime} \in \mathscr{L}^{2}\left(\frac{-\pi}{2 \sqrt{\beta}}, \frac{+\pi}{2 \sqrt{\beta}}\right) ;\right. \\
\left.\phi\left(\frac{+\pi}{2 \sqrt{\beta}}\right)=\phi\left(\frac{-\pi}{2 \sqrt{\beta}}\right)=0\right\},
\end{aligned}
$$

$$
\begin{aligned}
& \mathscr{D}\left(X^{\dagger}\right)=\left\{\psi, \psi^{\prime} \in \mathscr{L}^{2}\left(\frac{-\pi}{2 \sqrt{\beta}}, \frac{+\pi}{2 \sqrt{\beta}}\right)\right. \\
&\text { no other restriction on } \psi\}
\end{aligned}
$$

Therefore, $X$ is merely symmetric $\left(X=X^{\dagger}\right)$, but it is not a true self-adjoint operator. On the other hand, the momentum operator is a self-adjoint operator which can be shown using the von Neumann's theorem [11], that is, $P=P^{\dagger}$ and

$$
\mathscr{D}(P)=\mathscr{D}\left(P^{\dagger}\right)=\left\{\phi \in \mathscr{D}_{\max }(\mathbb{R})\right\},
$$

where $\mathscr{D}_{\max }$ denotes the maximal domain on which $P$ has a well-defined action

$$
\mathscr{D}_{\text {max }}(P)=\left\{\phi \in \mathscr{L}^{2}(\mathbb{R}): P \phi \in \mathscr{L}^{2}(\mathbb{R})\right\} .
$$

In this representation, the completeness relation and scalar product can be written as

$$
\begin{gathered}
\left\langle p^{\prime} \mid p\right\rangle=\delta\left(p-p^{\prime}\right), \\
\int_{-\pi / 2 \sqrt{\beta}}^{+\pi / 2 \sqrt{\beta}} \mathrm{d} p|p\rangle\langle p|=1 .
\end{gathered}
$$

Also the eigenfunctions of the position operator in momentum space are given by the solutions of the eigenvalue equation

$$
X u_{x}(p)=x u_{x}(p),
$$

where $u_{x}(p)=\langle p \mid x\rangle$. The normalized solution is

$$
u_{x}(p)=\sqrt{\frac{\sqrt{\beta}}{\pi}} \exp \left(-i \frac{p}{\hbar} x\right) .
$$

Note that the physical meaning of the present eigenstates is different from the ones provided in [11, 13]. Now using (10) we find the wave function in coordinate space as

$$
\psi(x)=\sqrt{\frac{\sqrt{\beta}}{\pi}} \int_{-\pi / 2 \sqrt{\beta}}^{+\pi / 2 \sqrt{\beta}} e^{i p x / \hbar} \phi(p) \mathrm{d} p .
$$

However, since the uncertainties for the eigenfunctions of the position operator are zero, that is, $\Delta X_{|x\rangle}=0,|x\rangle$ cannot be the physical solution. So, following Kempf et al. we define the maximal localization states $\left|\phi_{\xi}^{\mathrm{ML}}\right\rangle$ with the following properties [13]:

$$
\begin{gathered}
\left\langle\phi_{\xi}^{\mathrm{ML}}|X| \phi_{\xi}^{\mathrm{ML}}\right\rangle=\xi, \\
\Delta X_{\left|\phi_{\xi}^{\mathrm{ML}}\right\rangle}=(\Delta X)_{\min }=\hbar \sqrt{\beta} .
\end{gathered}
$$

These states also satisfy

$$
\left(X-\langle X\rangle+\frac{\langle[X, P]\rangle}{2(\Delta P)^{2}}(P-\langle P\rangle)\right)|\phi\rangle=0,
$$


where $\langle[X, P]\rangle=i \hbar\left(1+\beta(\Delta P)^{2}+\beta\langle P\rangle^{2}\right)$. Thus, in the momentum space the above equation takes the following form:

$$
\begin{aligned}
& {\left[i \hbar \frac{\partial}{\partial p}-\langle X\rangle\right.} \\
& \left.+i \hbar \frac{1+\beta(\Delta P)^{2}+\beta\langle P\rangle^{2}}{2(\Delta P)^{2}}\left(\frac{\tan (\sqrt{\beta} p)}{\sqrt{\beta}}-\langle P\rangle\right)\right] \phi(p)=0,
\end{aligned}
$$

which has the solution

$$
\begin{aligned}
\phi(p)=\mathcal{N} \exp & {\left[\left(-\frac{i}{\hbar}\langle X\rangle+\frac{1+\beta(\Delta P)^{2}+\beta\langle P\rangle^{2}}{2(\Delta P)^{2}}\langle P\rangle\right) p\right.} \\
+ & \left.\left(\frac{1+\beta(\Delta P)^{2}+\beta\langle P\rangle^{2}}{2(\Delta P)^{2}}\right) \frac{\ln [\cos (\sqrt{\beta} p)]}{\beta}\right] .
\end{aligned}
$$

To find the absolutely maximal localization states we need to choose the critical momentum uncertainty $\Delta P=1 / \sqrt{\beta}$ that gives the minimal length uncertainty and takes $\langle P\rangle=0$; that is,

$$
\phi_{\xi}^{\mathrm{ML}}(p)=\mathcal{N} \cos (\sqrt{\beta} p) e^{-i p \xi / \hbar},
$$

where the normalization factor is given by

$$
\mathcal{N}=\sqrt{\frac{2 \sqrt{\beta}}{\pi}} .
$$

It is straightforward to check that $\phi_{\xi}^{\mathrm{ML}}(p)$ exactly satisfies (14) and (15). Because of the fuzziness of space, these maximal localization states are not mutually orthogonal:

$$
\begin{aligned}
\left\langle\phi_{\xi^{\prime}}^{\mathrm{ML}} \mid \phi_{\xi}^{\mathrm{ML}}\right\rangle & =\mathcal{N}^{2} \int_{-\pi / 2 \sqrt{\beta}}^{+\pi / 2 \sqrt{\beta}} \mathrm{d} p \cos ^{2}(\sqrt{\beta} p) e^{-i p\left(\xi-\xi^{\prime}\right) / \hbar} \\
& =\frac{8 \beta^{3 / 2} \hbar^{3}}{\pi} \frac{\sin \left[\pi\left(\xi-\xi^{\prime}\right) / 2 \hbar \sqrt{\beta}\right]}{\left(\xi-\xi^{\prime}\right)^{3}-4 \beta \hbar^{2}\left(\xi-\xi^{\prime}\right)} .
\end{aligned}
$$

To find the quasiposition wave function $\chi(\xi)$, we define

$$
\chi(\xi) \equiv\left\langle\phi_{\xi}^{\mathrm{ML}} \mid \phi\right\rangle,
$$

where in the limit $\beta \rightarrow 0$ it goes to the ordinary position wave function $\chi(\xi)=\langle\xi \mid \phi\rangle$. Now the transformation of the wave function in the momentum representation into its counterpart quasiposition wave function is

$$
\begin{aligned}
\chi(\xi) & =\mathcal{N} \int_{-\pi / 2 \sqrt{\beta}}^{+\pi / 2 \sqrt{\beta}} \mathrm{d} p \cos (\sqrt{\beta} p) e^{i p \xi / \hbar} \phi(p), \\
& =\frac{1}{\sqrt{2}}[\psi(\xi+\hbar \sqrt{\beta})+\psi(\xi-\hbar \sqrt{\beta})] .
\end{aligned}
$$

So the quasiposition wave function at $\langle X\rangle=\xi$ is the superposition of the coordinate space wave functions at $\xi+\hbar \sqrt{\beta}$ and $\xi-\hbar \sqrt{\beta}$. In other words, the quasiposition wave function is the result of the interference of two coordinate space wave functions.

\section{Boundary Conditions}

In this section, we discuss how the boundary conditions in quasiposition space can be determined by fixing the boundary conditions in coordinate space.

3.1. Dirichlet Boundary Condition. Consider the following Dirichlet boundary condition in coordinate space

$$
\left.\psi(x)\right|_{x=\xi_{0}}=0,
$$

which gives

$$
\int_{-\pi / 2 \sqrt{\beta}}^{+\pi / 2 \sqrt{\beta}} e^{i(p / \hbar) \xi_{0}} \phi(p) \mathrm{d} p=0 .
$$

Now, using (24) and to first of the GUP parameter, we find

$$
\left.\chi(\xi)\right|_{\xi=\xi_{0}}=\frac{\hbar^{2} \beta}{\sqrt{2}} \psi^{\prime \prime}\left(\xi_{0}\right)+\mathcal{O}\left(\beta^{2}\right)
$$

which fixes the quasiposition wave functions at $\xi=\xi_{0}$. Note that, for the following class of the coordinate space solutions

$$
\psi(x)=\left\{\begin{array}{l}
A \sin (\omega x)+B \cos (\omega x), \\
\sin ^{n}(\omega x), \\
\cos ^{n}(\omega x),
\end{array}\right.
$$

we exactly have

$$
\left.\chi(\xi)\right|_{\xi=\xi_{0}}=\left.\psi(x)\right|_{x=\xi_{0}}=0,
$$

where $\xi_{0}$ are the zeros of (28). So, for these cases, the quasiposition wave functions obey the same boundary conditions as coordinate space wave functions.

3.2. Neumann Boundary Condition. The Neumann boundary condition determines the values that the derivative of a wave function is to take on the boundary of the domain. Let us consider the following boundary condition in coordinate space:

$$
\left.\psi^{\prime}(x)\right|_{x=\xi_{0}}=0
$$

where prime denotes the derivation with respect to the argument. This equation is equivalent to

$$
\int_{-\pi / 2 \sqrt{\beta}}^{+\pi / 2 \sqrt{\beta}} e^{i(p / \hbar) \xi_{0}} p \phi(p) \mathrm{d} p=0 .
$$

Also, using (23) we have

$$
\chi^{\prime}(\xi)=\frac{1}{\sqrt{2}}\left[\psi^{\prime}(\xi+\hbar \sqrt{\beta})+\psi^{\prime}(\xi-\hbar \sqrt{\beta})\right] .
$$

Therefore, to first order we obtain

$$
\left.\chi^{\prime}(\xi)\right|_{\xi=\xi_{0}}=\frac{\hbar^{2} \beta}{\sqrt{2}} \psi^{\prime \prime \prime}\left(\xi_{0}\right)+\mathcal{O}\left(\beta^{2}\right) .
$$


Moreover, for the coordinate space wave functions presented in (28) we exactly find

$$
\left.\chi^{\prime}(\xi)\right|_{\xi=\xi_{0}}=\left.\psi^{\prime}(x)\right|_{x=\xi_{0}}=0 .
$$

So, both $\chi(\xi)$ and $\psi(x)$ satisfy the same Neumann boundary condition.

Now let us elaborate on the correspondence between the uncertainties in position and the imposition of localized boundary conditions. In the GUP framework, it is not possible to measure the position of a particle more accurate than $(\Delta X)_{\min }$. So we cannot define the potentials with infinitely sharp boundaries. In fact, the position of these boundaries can be only specified within this uncertainty. As it is shown in [11], the potentials with infinitely sharp boundaries such as the particle in a box potential cannot be properly defined in the GUP framework with respect to ordinary quantum mechanics.

\section{Conclusions}

In this paper, we have investigated the issue of the boundary conditions in deformed quantum mechanics which implies a minimal length uncertainty proportional to the Planck length. We found the coordinate space wave functions, maximal localization states, and quasiposition wave functions using a formally self-adjoint representation. We indicated that the position operator $X$ is merely symmetric and the momentum operator $P$ is truly self-adjoint which agrees with [13]. The maximal localization states are the physical states and obey the minimal length uncertainty; that is, $\Delta X_{\left|\phi_{\xi}^{\mathrm{ML}}\right\rangle}=$ $\hbar \sqrt{\beta}$. We showed that the boundary conditions in coordinate space specify the boundary conditions in quasiposition space and found the exact relations for both Dirichlet and Neumann boundary conditions. Also, for a particular class of solutions, the boundary conditions are found to be the same in coordinate and quasiposition spaces. In fact, because of (15) the quasiposition wave functions $\left\langle\phi_{\xi}^{\mathrm{ML}} \mid \phi\right\rangle$ and their boundary conditions contain the standard physical interpretation.

\section{Acknowledgment}

The authors would like to thank the referees for invaluable comments and important suggestions which considerably improved the quality of the paper.

\section{References}

[1] S. Hossenfelder, "Minimal length scale scenarios for quantum gravity," Reviews in Relativity, vol. 16, no. 2, 2013.

[2] D. Amati, M. Ciafaloni, and G. Veneziano, "Can spacetime be probed below the string size?" Physics Letters B, vol. 216, no. 1-2, pp. 41-47, 1989.

[3] K. Konishi, G. Paffuti, and P. Provero, "Minimum physical length and the generalized uncertainty principle in string theory," Physics Letters. B, vol. 234, no. 3, pp. 276-284, 1990.

[4] M. Maggiore, "A generalized uncertainty principle in quantum gravity," Physics Letters B, vol. 304, no. 1-2, pp. 65-69, 1993.
[5] F. Scardigli, "Generalized uncertainty principle in quantum gravity from micro-black hole gedanken experiment," Physics Letters B, vol. 452, no. 1-2, pp. 39-44, 1999.

[6] M. Maggiore, "Quantum groups, gravity, and the generalized uncertainty principle," Physical Review D, vol. 49, no. 10, pp. 5182-5187, 1994.

[7] S. Ghosh and S. Mignemi, "Quantum mechanics in de Sitter space," International Journal of Theoretical Physics, vol. 50, no. 6, pp. 1803-1808, 2011.

[8] C. Castro, "String theory, scale relativity and the generalized uncertainty principle," Foundations of Physics Letters, vol. 10, no. 3, pp. 273-293, 1997.

[9] S. Hossenfelder, M. Bleicher, S. Hofmann, J. Ruppert, S. Scherer, and H. Stöcker, "Signatures in the Planck regime," Physics Letters $B$, vol. 575 , no. 1-2, pp. 85-99, 2003.

[10] C. Bambi and F. R. Urban, "Natural extension of the generalized uncertainty principle," Classical and Quantum Gravity, vol. 25, no. 9, Article ID 095006, 2008.

[11] P. Pedram, "New approach to nonperturbative quantum mechanics with minimal length uncertainty," Physical Review $D$, vol. 85, Article ID 024016, 2012.

[12] S. Das and E. C. Vagenas, "Universality of quantum gravity corrections," Physical Review Letters, vol. 101, Article ID 221301, 2008.

[13] A. Kempf, G. Mangano, and R. B. Mann, "Hilbert space representation of the minimal length uncertainty relation," Physical Review D, vol. 52, no. 2, pp. 1108-1118, 1995.

[14] D. J. Gross and P. F. Mende, "String theory beyond the Planck scale," Nuclear Physics. B, vol. 303, no. 3, pp. 407-454, 1988.

[15] C. A. Mead, "Possible connection between gravitation and fundamental length," vol. 135, pp. B849-B862, 1964.

[16] H. Salecker and E. P. Wigner, "Quantum limitations of the measurement of space-time distances," vol. 109, pp. 571-577, 1958.

[17] T. Padmanabhan, "Physical significance of Planck length," Annals of Physics, vol. 165, no. 1, pp. 38-58, 1985.

[18] T. Padmanabhan, "Planck length as the lower bound to all physical length scales," General Relativity and Gravitation, vol. 17, no. 3, pp. 215-221, 1985.

[19] G. t. Hooft and M. Veltman, "One-loop divergencies in the theory of gravitation," AnnaLes de L'Institut Henri Poincaré A, vol. 20 , no. $69,1974$.

[20] R. J. Adler and D. I. Santiago, "On gravity and the uncertainty principle," Modern Physics Letters A, vol. 14, no. 20, article 1371, 1999.

[21] I. Pikovski, M. R. Vanner, M. Aspelmeyer, M. S. Kim, and Č. Brukner, "Probing planck-scale physics with quantum optics," Nature Physics, vol. 8, no. 5, pp. 393-397, 2012.

[22] G. Amelino-Camelia, J. Ellis, N. E. Mavromatos, D. V. Nanopoulos, and S. Sarkar, "Tests of quantum gravity from observations of $\gamma$-ray bursts," Nature, vol. 393, pp. 763-765, 1998.

[23] U. Jacob and T. Piran, "Neutrinos from gamma-ray bursts as a tool to explore quantum-gravity-induced Lorentz violation," Nature Physics, vol. 7, pp. 87-90, 2007. 

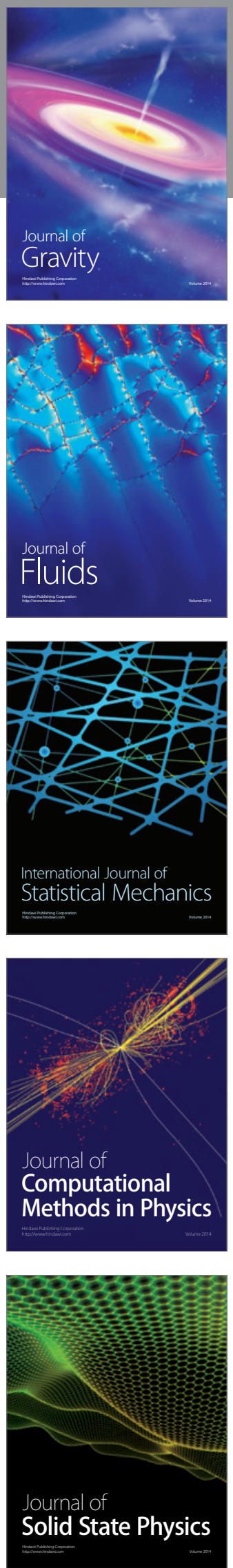

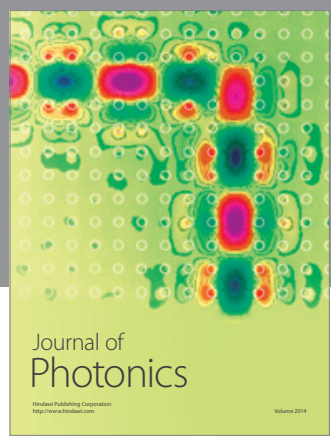

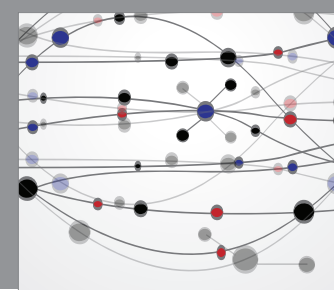

The Scientific World Journal

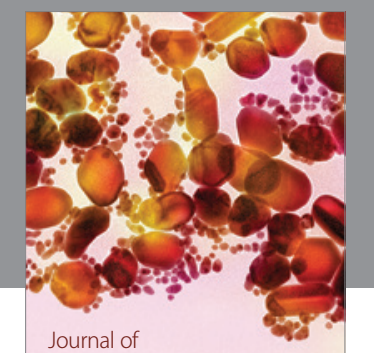

Soft Matter
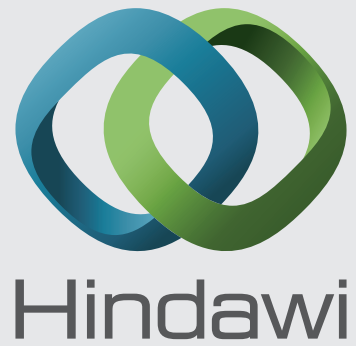

Submit your manuscripts at

http://www.hindawi.com
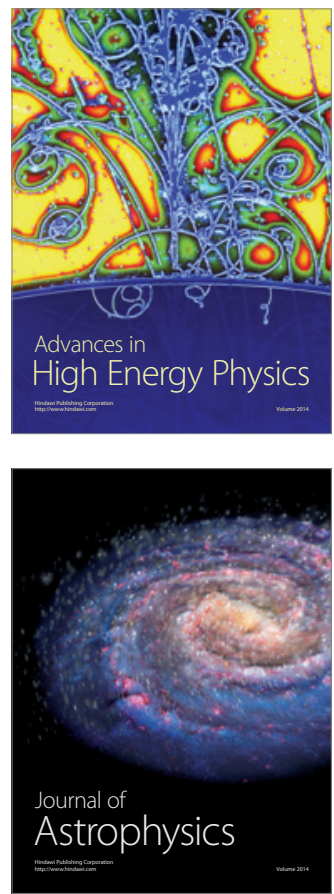
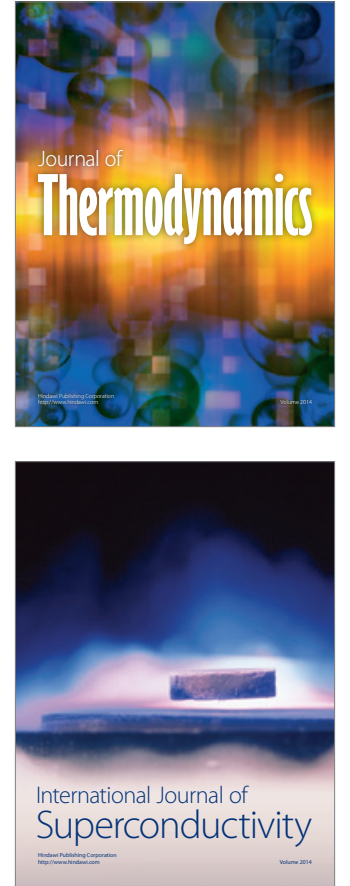
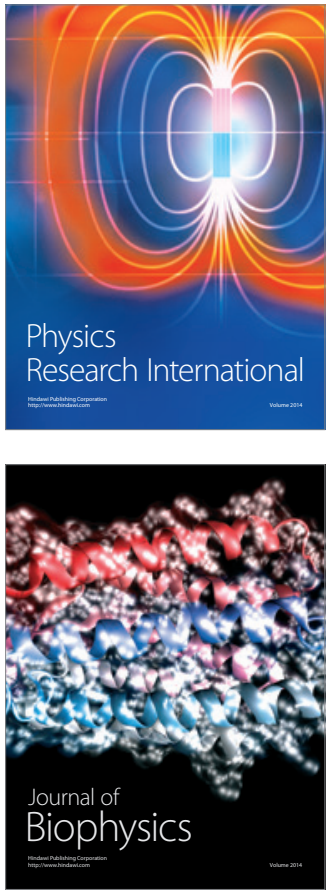
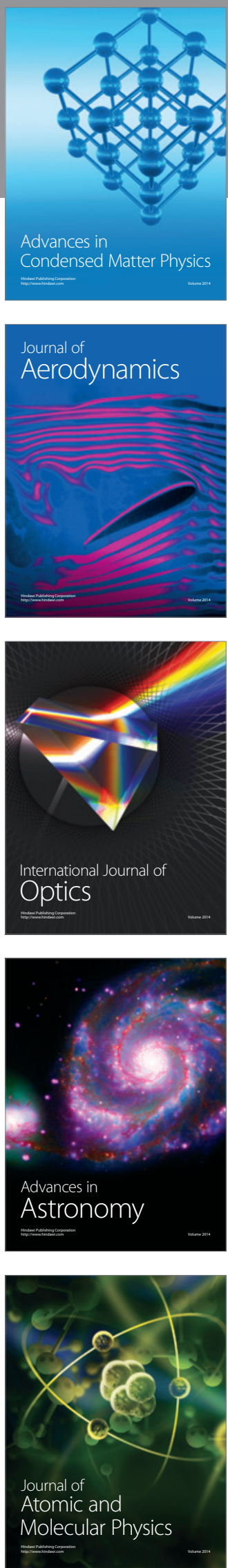